\title{
Micropapillary and solid subtypes of invasive lung adenocarcinoma: Clinical predictors of histopathology and outcome
}

\author{
Min Jae Cha, MD, ${ }^{\mathrm{a}}$ Ho Yun Lee, MD, ${ }^{\mathrm{a}}$ Kyung Soo Lee, MD, ${ }^{\mathrm{a}} \mathrm{Ji}$ Yun Jeong, MD, ${ }^{\mathrm{b}}$ Joungho Han, MD, \\ Young Mog Shim, MD, ${ }^{\mathrm{c}}$ and Hye Sun Hwang, $\mathrm{MD}^{\mathrm{a}}$
}

Objective: To evaluate the clinical effect of the presence of a micropapillary or solid subtype on the outcomes in lung adenocarcinoma and to determine the predictors of such a histopathologic diagnosis.

\begin{abstract}
Methods: A total of 511 patients with lung adenocarcinoma $\leq 3 \mathrm{~cm}$ were included. According to the presence of micropapillary or solid subtypes, we classified the patients into 4 subgroups: both subtypes absent $(\mathrm{MP}-/ \mathrm{S}-$, $\mathrm{n}=87$ ), either subtype present $(\mathrm{MP}+/ \mathrm{S}-, \mathrm{n}=207$ and $\mathrm{MP}-/ \mathrm{S}+, \mathrm{n}=196)$, and both present $(\mathrm{MP}+/ \mathrm{S}+$, $\mathrm{n}=21$ ) to determine the association between the micropapillary or solid subtype and survival outcome or clinical and imaging conditions. Univariate and multivariate analyses were undertaken to determine the parameters, allowing the prediction of the presence of the micropapillary or solid subtype.
\end{abstract}

Results: Overall survival (OS) and disease-free survival (DFS) differed significantly among the 4 subgroups $(P<.001$ and $P=.004$, respectively). The MP-/S - tumors showed better DFS than those containing either the micropapillary or solid subtype. Patients with the micropapillary subtype had significantly worse OS than patients without the micropapillary subtype. This difference remained significant, together with stage, after adjustment for gender, age, adjuvant therapy, tumor size, and solid subtype (DFS and OS, $P=.016$ and $P=.002$, respectively). On multivariate analysis, greater than stage I, tumor size $\geq 2.5 \mathrm{~cm}$, solid mass, and maximal standardized uptake value of $\geq 7$ were independent predictors of the presence of a micropapillary or solid subtype.

Conclusions: Micropapillary and solid subtypes are common in tumors greater than stage I, with size $\geq 2.5 \mathrm{~cm}$, pure solid type, and maximal standardized uptake value of $\geq 7$, which were predictors for poor DFS. The presence of the micropapillary subtype was a single prognostic factor for OS. (J Thorac Cardiovasc Surg 2014;147:921-8)

Supplemental material is available online.

Earn CME credits at

http://cme.ctsnetjournals.org

With advances in the understanding of lung adenocarcinoma (ADC), a new classification was published by the International Association for the Study of Lung Cancer (IASLC), American Thoracic Society (ATS), and European Respiratory Society (ERS) in 2011. ${ }^{1}$ In this novel proposal,

\footnotetext{
From the Department of Radiology and Center for Imaging Science, ${ }^{\mathrm{a}}$ Department of Pathology, ${ }^{\mathrm{b}}$ and Department of Thoracic Surgery, ${ }^{\mathrm{c}}$ Samsung Medical Center, Sungkyunkwan University School of Medicine, Seoul, Korea.

Disclosures: Authors have nothing to disclose with regard to commercial support.

Dr Jeong is currently with the Department of Pathology, Kyungpook National Univer-

sity Hospital, Kyungpook National University School of Medicine, Daegu, Korea.

Received for publication May 15, 2013; revisions received Sept 5, 2013; accepted for

publication Sept 19, 2013; available ahead of print Nov 6, 2013.

Address for reprints: Ho Yun Lee, MD, Department of Radiology, Samsung Medical Center, Sungkyunkwan University School of Medicine, 50 Ilwon-Dong, Kangnam$\mathrm{Ku}$, Seoul 135-710 Korea (E-mail: hoyunlee96@gmail.com).

$0022-5223 / \$ 36.00$

Copyright (c) 2014 by The American Association for Thoracic Surgery

http://dx.doi.org/10.1016/j.jtcvs.2013.09.045
}

they defined 5 distinctive subtypes of invasive lung ADCs in association with the prognosis, stating lepidic as favorable, acinar and papillary as intermediate, and micropapillary and solid as poor. In particular, micropapillary, which was not included in the 2004 World Health Organization classification, was added, and the clinical effect of this new subtype is one of the academic issues.

To date, several studies have attempted to determine the prognostic value of the histologic subtypes of the new lung ADC classification. ${ }^{2-5}$ From these previous studies, we hypothesized that additional work about the predictive value of the histologic subtypes according to the IASLC/ ATS/ERS proposal, ${ }^{1}$ especially the micropapillary and solid patterns as poor prognostic factors, would improve the clinical relevance of this novel classification. In addition, clinical and radiologic parameters that can allow one to suggest the presence of a micropapillary or solid subtype would help predict the prognosis of patients with invasive ADC preoperatively. ${ }^{6}$ In the present study, we investigated the effect of the presence of a micropapillary or solid pattern on overall survival (OS) and disease-free survival (DFS) in patients with invasive pulmonary ADC of all tumor stages. We also performed analyses to determine the clinical and radiologic predictors that could provide suggestions regarding the presence of a histopathologically proven micropapillary or solid pattern of ADC. 


\section{Abbreviations and Acronyms \\ ADC = adenocarcinoma \\ ATS $=$ American Thoracic Society \\ $\mathrm{CI}=$ confidence interval \\ $\mathrm{CT}=$ computed tomography \\ DFS $=$ disease-free survival \\ ERS = European Respiratory Society \\ GGO $=$ ground glass opacity \\ IASLC $=$ International Association for the Study of Lung Cancer \\ $\mathrm{MP}=$ micropapillary \\ $\mathrm{OR} \quad=$ odds ratio \\ OS = overall survival \\ PET = positron emission tomography \\ ROC = receiver operating characteristic \\ $\mathrm{S} \quad=$ solid \\ SUVmax $=$ maximal standardized uptake value}

\section{METHODS}

The institutional review board approved the present study (approval no. 2008-10-057), and informed consent was waived for the use of the patients' medical data.

\section{Patients}

Using an oncology database at Samsung Medical Center from September 2003 to August 2011, we identified 621 patients with completely resected solitary invasive lung ADC that was $\leq 3 \mathrm{~cm}$. None had a history of neoadjuvant treatment. The diagnoses were made according to the criteria of the current World Health Organization classification for lung cancer. ${ }^{7}$ Of the 621 patients, 68 were excluded because of prognosis-related factors: the presence of micrometastasis at surgery in 25 and the concomitancy of other cancer in 43 . Another 42 patients were excluded because of radiology- or pathologically related factors: (1) insufficient pathologic slides for evaluation of the whole tumor $(n=26)$; (2) poor computed tomography $(\mathrm{CT})$ image quality $(\mathrm{n}=10)$; and (3) limited tumor evaluation owing to combined extensive inflammation or infarction $(n=6)$.

Thus, 511 patients ( 279 males, 232 females; median age, 61 years) were included in the present analysis (Figure 1). The median follow-up period was 77 months (range, 10.1-255.8). First, the patients were classified by the most predominant subtypes as follows: $49(10 \%)$ with lepidicpredominant tumors, $267(52 \%)$ with acinar-predominant tumors, 61 $(12 \%)$ with papillary-predominant tumors, $107(21 \%)$ with solidpredominant tumors, and $27(5 \%)$ with micropapillary-predominant tumors. Next, we classified patients into 4 subgroups according to the presence of the micropapillary and solid subtypes: both subtypes absent (MP-/S-), micropapillary subtype present and solid subtype absent $(\mathrm{MP}+/ \mathrm{S}-)$, micropapillary subtype absent and solid subtype present $(\mathrm{MP}-/ \mathrm{S}+)$, and both present $(\mathrm{MP}+/ \mathrm{S}+)$. Such subtyping was conducted to determine the association between the micropapillary and/or solid subtype and OS and DFS and the clinical and radiologic findings. Of the 511 ADCs, 87 were classified as MP-/S - (17.0\%), 207 as MP+/S- $(40.5 \%)$, 196 as MP-/S+ (38.4\%), and 21 as MP+/S+ (4.1\%). Finally, to investigate the clinical and radiologic parameters predicting the presence of the micropapillary or solid subtype, we divided the patients into 2 groups: those with tumors containing either the micropapillary or solid subtype $(n=424)$ and those without tumors containing either subtype $(n=87)$.

\section{Clinical Assessment}

The clinical information was available from the patient medical records. We screened the data, including gender, age, tumor stage using the TNM classification, resection type, adjuvant therapy, survival, and disease progression. OS was defined as the interval from surgery to the date of death or final follow-up visit. DFS was defined as the interval from surgery to the point of any definite clinical or pathologic evidence of local or distant disease recurrence or last evaluation.

\section{Imaging and Interpretation}

The imaging characteristics of each primary lung tumor were evaluated using chest $\mathrm{CT}$ and the positron emission tomography (PET) component images of PET-CT (see Appendix E1). PET-CT and chest CT were performed within the 2-week period from surgery. The imaging methods have been described in detail in previous reports. 2,8

Two radiologists (E.J. L. and H.S.H., with 5 and 7 years of experience in chest radiology, respectively), who were unaware of the clinical information, independently evaluated the CT scans. The CT scans were retrieved using the Picture Archiving and Communications System (Centricity, GE Healthcare, General Electric, Fairfield, Conn). The radiologic parameters, including tumor size, lesion density, tumor solidity, margin status, and the presence of spiculations or lobulations were recorded. The largest diameter measurements were obtained manually using the Picture Archiving and Communications System measurement electronic tool in all cases. For measurements of lesion density, the region of interest covering each target lesion was drawn on the axial postcontrast-enhanced scan as large as possible (at least two thirds of the longest diameter). The mean CT attenuation value with standard deviation was measured in Hounsfield units. Tumor solidity was categorized as solid, partly solid, and ground glass opacity (GGO). GGO on the CT scans was defined as a hazy increase in lung density without obscuration of the pulmonary vessels. The tumor margin was classified as well-defined or ill-defined. Spiculation or lobulation was defined as a radiologic outline of the lesion showing sharp points or smooth protrusions into the surrounding tissue, respectively.

All PET images were reviewed by an experienced nuclear medicine physician (B.T.K., with 13 years of experience in PET-CT interpretation). The fluorodeoxyglucose uptake of the primary cancer was quantified by calculating the maximal standardized uptake value (SUVmax) using PET region-of-interest analysis.

\section{Pathologic Evaluation}

Whole tumor tissue sections were obtained, and each was placed on a slide. Comprehensive histologic subtyping was performed by 2 pathologists (J.H.H. and J.Y.J., with 18 and 5 years of experience in pulmonary pathology, respectively), together at a multihead microscope, and discussed until consensus was achieved. The tumors were divided into 6 distinctive subtypes according to the IASLC/ATS/ERS classification scheme as (1) lepidic, (2) acinar, (3) papillary, (4) micropapillary, (5) solid, and (6) a variant, including mucinous (Figure E1). For each case, histologic subtyping was performed for the primary tumor in a semiquantitative manner, to the nearest $5 \%$ level, summing to a total of $100 \%$ subtype components per tumor. For the micropapillary and solid subtypes, we also considered the micropapillary or solid subtype to be present when the subtype occupied $\geq 1 \%$ of the entire tumor. ${ }^{9}$ Next, the most predominant pattern in a mixed-type ADC was defined as the histopathologic subtype that constituted the greatest percentage of the tumor. The lowest limit for the predominant subtype was set at $30 \%$, as previously described. ${ }^{1}$

\section{Statistical Analysis}

The clinical prognostic parameters of each subgroup were compared using 1-way analysis of variance with the post hoc test of Bonferroni. The DFS and OS were estimated using the Kaplan-Meier method, and the log-rank test was used to evaluate the differences among the subgroups. 


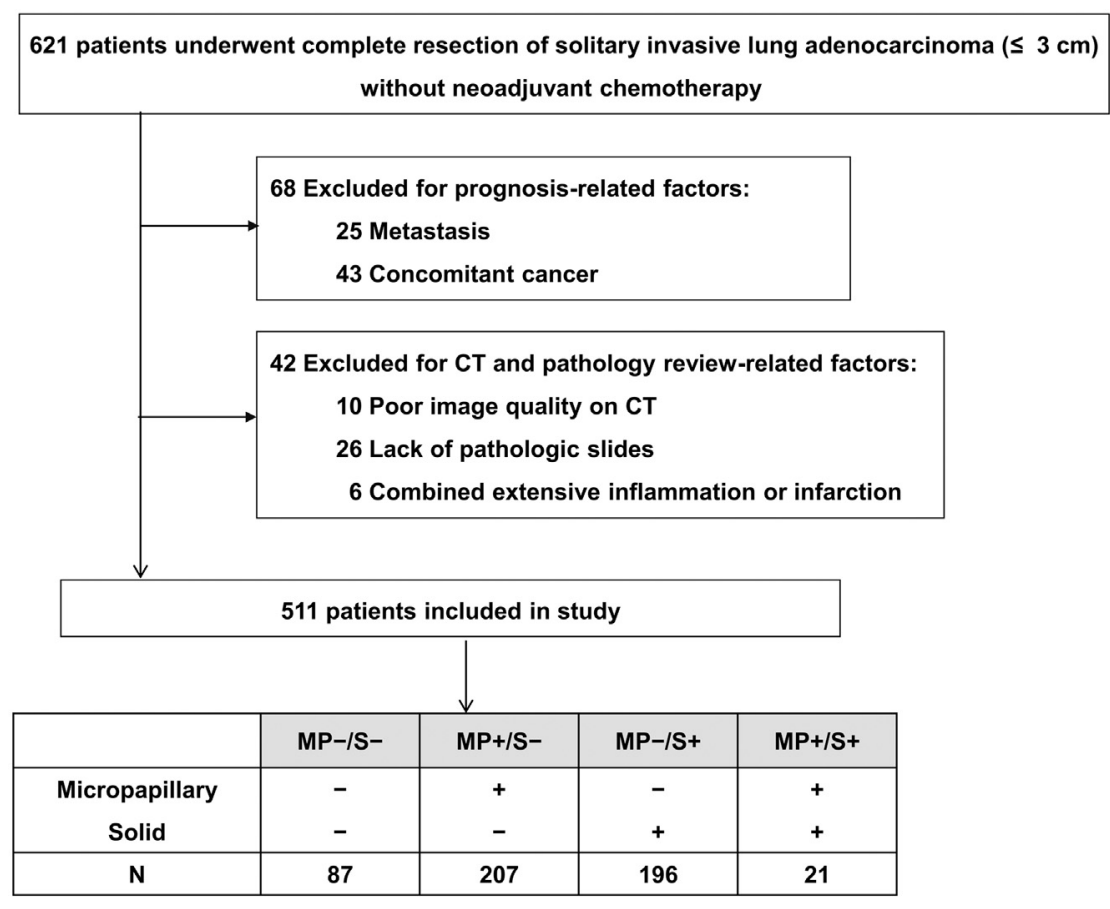

FIGURE 1. Flow chart of patient enrollment. $C T$, Computed tomography; $M P$, micropapillary; $S$, solid.

Multivariate analysis was used to assess the effect of the covariates on DFS and OS and was performed using backward Cox proportional hazards regression analysis with a step-down method. The variables with $P<.10$ on univariate analysis were used as the input variables for the multivariate analysis.

Univariate and multivariate analyses were undertaken to assess the clinical and imaging predictors for the presence of a micropapillary or solid subtype. The optimal cutoff values for tumor size and SUVmax were calculated using receiver operating characteristic (ROC) curve analysis. The optimal cutoff values were determined as the point closest to the upper left hand corner of the ROC curve.

As a supplementary analysis, we constructed ROC curves, plotting the sensitivity versus 1 minus specificity, to evaluate the differentiating performance of multivariate logistic regression models in discriminating the presence of a micropapillary or solid pattern. The area under the ROC curve, a measure of predictive power, was calculated. The statistical analysis was performed using the Statistical Package for Social Sciences, version 15, statistical software for Windows (SPSS Inc, Chicago, Ill). $P \leq .05$ was considered statistically significant.

\section{RESULTS}

\section{Correlation of Pathologic Subgroups With Clinical Prognostic Parameters}

Reclassification of the 511 ADC specimens resulted in 87 with MP-/S - (17.0\%), 207 with MP+/S- $40.5 \%), 196$ with $\mathrm{MP}-/ \mathrm{S}+(38.4 \%)$, and 21 with $\mathrm{MP}+/ \mathrm{S}+(4.1 \%$; Table 1). A total of 20 ADCs had a mucinous component $(\mathrm{n}=19)$ or fetal component $(\mathrm{n}=1)$; however, they were not most predominant. The median percentage of micropapillary involvement in 228 patients was $2.5 \%$ (interquartile range, $9 \% ; 25$ th percentile, $1 \% ; 75$ th percentile, $10 \%$ ). The median percentage of solid involvement in 217 patients was
$25 \%$ (interquartile range, $15 \% ; 25$ th percentile, $20 \% ; 75$ th percentile, $35 \%$ ).

The 4 subgroups were significantly different in terms of gender $(P=.01)$, tumor size (median, $27.3 \mathrm{~mm} ; P<.01$ ), TNM stage $(P<.01)$, necessity of adjuvant chemotherapy $(P=.01)$, disease progression (including local recurrence and metastasis; $P=.01)$, and death rate $(P=.03$; Table 1). Tumors without the micropapillary or solid subtype (MP-/S-) tended to be smaller, less invasive (as determined by the tumor stage), less frequently required adjuvant chemotherapy, and less frequently resulted in disease progression or death compared with those containing either the micropapillary or solid subtype. In addition, female patients were more likely to have $\mathrm{MP}-/ \mathrm{S}-$ tumors.

\section{Survival Analyses in Association With Histologic Subtypes}

First, we performed a survival analysis according to the "most predominant subtypes," as proposed by the IASLC/ATS/ERS. DFS and OS differed significantly among the 5 different predominant subtype tumors $(P<.001$ and $P=.042$, respectively; Figure 2$)$. The patients with lepidic-predominant tumors had a good prognosis but those with solid- and micropapillary-predominant tumors had a poor prognosis.

Thus, we determined whether the presence of a micropapillary or solid subtype, merely $1 \%$ of the entire tumor, can affect the survival outcome (Figure 3, $A$ and $B$ ). OS and 
TABLE 1. Clinical and prognostic characteristics of 511 ADC specimens in association with 4 pathologic subgroups

\begin{tabular}{|c|c|c|c|c|c|c|}
\hline Characteristic & Total & $\mathbf{M P}-/ \mathbf{S}-(\mathbf{n}=\mathbf{8 7})$ & $\mathbf{M P}+/ \mathbf{S}-(\mathbf{n}=\mathbf{2 0 7})$ & $M P-/ S+(n=196)$ & $\mathbf{M P}+/ \mathbf{S}+(\mathbf{n}=\mathbf{2 1})$ & $P$ value \\
\hline Gender (n) & & & & & & .01 \\
\hline Male & $279(55)$ & $38(44)$ & $105(52)$ & $123(63)$ & $13(62)$ & \\
\hline Female & $232(45)$ & $49(56)$ & $102(49)$ & $73(37)$ & $8(38)$ & \\
\hline Age (y) & & & & & & .46 \\
\hline Range & $38-86$ & $39-81$ & $42-86$ & $38-81$ & $42-80$ & \\
\hline Median & 61 & 61 & 61 & 61 & 61 & \\
\hline Tumor size (mm) & & & & & & $<.01$ \\
\hline Range & $7-30$ & $9-30$ & $7-30$ & $8-30$ & $14-30$ & \\
\hline Median & 27.3 & 21.3 & 27.6 & 27.7 & 27.8 & \\
\hline Stage (n) & & & & & & $<.01$ \\
\hline I & $321(63)$ & $80(92)$ & $124(60)$ & $106(54)$ & $11(52)$ & \\
\hline II & $104(20)$ & $6(7)$ & $42(20)$ & $51(26)$ & $5(24)$ & \\
\hline IIIA & $86(17)$ & $1(1)$ & $41(20)$ & $39(20)$ & $5(24)$ & \\
\hline pLN status (n) & & & & & & $<.01$ \\
\hline No & $358(70)$ & $81(93)$ & $137(66)$ & $129(66)$ & $11(52)$ & \\
\hline N1 & $84(16)$ & $5(7)$ & $39(19)$ & $34(17)$ & $6(29)$ & \\
\hline $\mathrm{N} 2$ & $69(14)$ & $1(0)$ & $31(15)$ & $33(17)$ & $4(19)$ & \\
\hline Resection extent (n) & & & & & & .74 \\
\hline Segmentectomy & $34(7)$ & $8(9)$ & $11(5)$ & $14(7)$ & $1(5)$ & \\
\hline Lobectomy & $469(92)$ & $77(89)$ & $195(94)$ & $177(91)$ & $20(95)$ & \\
\hline Pneumonectomy & $8(1)$ & $2(2)$ & $1(1)$ & $5(2)$ & $0(0)$ & \\
\hline Most predominant histologic subtype (n) & & & & & & $<.01$ \\
\hline Lepidic & $49(10)$ & $30(36)$ & $15(7)$ & $3(1)$ & $0(0)$ & \\
\hline Acinar & $267(52)$ & $52(58)$ & $126(61)$ & $76(39)$ & $15(70)$ & \\
\hline Papillary & $61(12)$ & $5(6)$ & $41(20)$ & $13(6)$ & $2(10)$ & \\
\hline Solid & $107(21)$ & $0(0)$ & $0(0)$ & $105(54)$ & $2(10)$ & \\
\hline Micropapillary & $27(5)$ & $0(0)$ & $25(12)$ & $0(0)$ & $2(10)$ & \\
\hline Adjuvant chemotherapy (n) & $70(14)$ & $2(2)$ & $31(15)$ & $33(17)$ & $4(19)$ & .01 \\
\hline Disease progression (n) & $146(29)$ & $12(14)$ & $63(30)$ & $58(30)$ & $13(62)$ & .01 \\
\hline Local recurrence & $11(2)$ & $1(1)$ & $4(2)$ & $3(3)$ & $3(14)$ & \\
\hline Metastasis & $135(27)$ & $11(13)$ & $59(28)$ & $55(28)$ & $10(48)$ & \\
\hline Death (n) & $22(4)$ & $3(3)$ & $12(6)$ & $4(2)$ & $3(14)$ & .03 \\
\hline
\end{tabular}

Percentages refer to each individual characteristic, with the total $100 \%$ per group. $M P-/ S-$, Tumors without micropapillary or solid subtype; $M P+/ S-$, any micropapillary but no solid subtype; $M P-/ S+$, any solid but no micropapillary subtype; $M P+/ S+$, both micropapillary and solid subtypes; $p L N$, pathologic lymph node.

DFS differed significantly among the 4 subgroups $(P<.01$ for all). Those with MP-/S - tumors had better DFS than those with either the micropapillary or solid subtype. In contrast, those with MP+/S+ tumors had poorer OS than the other groups. Survival analyses of the tumors with versus without a micropapillary subtype also demonstrated a significant difference in DFS and OS $(P=.02$ and $P<.01$, respectively; Figure 3, $C$ and $D$ ).

When we analyzed the survival rates of the patients with tumors with and without a solid subtype, however, no significant difference was found in OS $(P=.87)$. Only DFS was significantly worse for patients with tumors having a solid subtype $(P<.01$; Figure $3, E$ and $F)$. Therefore, we performed a subgroup analysis of OS, focusing on the presence of the micropapillary subtype only. In the subgroup with the micropapillary subtype, the solid subtype played a role as a strong predictor for determining OS. However, without the micropapillary subtype, the solid subtype showed little influence on OS
(Figure E2). This implies that micropapillary subtype is a single risk factor for predicting a poor prognosis and that the validity of the solid subtype as a predictive factor has not yet been proved. The solid pattern contributed to the prognosis only when it coexisted with the micropapillary subtype.

Finally, multivariate analysis verified that the tumor stage and the presence of the micropapillary subtype were the $2 \mathrm{in}$ dependent factors significantly associated with OS (Table 2). We observed that patients with stage I cancer showed greater OS rates than those with stage II and III (hazard ratio, $0.15 ; 95 \%$ confidence interval $[\mathrm{CI}], 0.03-0.84 ; P=.03)$. In addition, patients with the micropapillary subtype had lower survival rates than those without the micropapillary subtype (HR, 4.17; 95\% CI, 1.28-13.63; $P=.02$ ). However, we did not observe a significant association between the solid subtype and $\operatorname{OS}(P=.33)$. The association was also not significant for tumor size $(P=.08)$, gender $(P=.33)$, age $(P=.06)$, or use of adjuvant chemotherapy $(P=.82)$. 

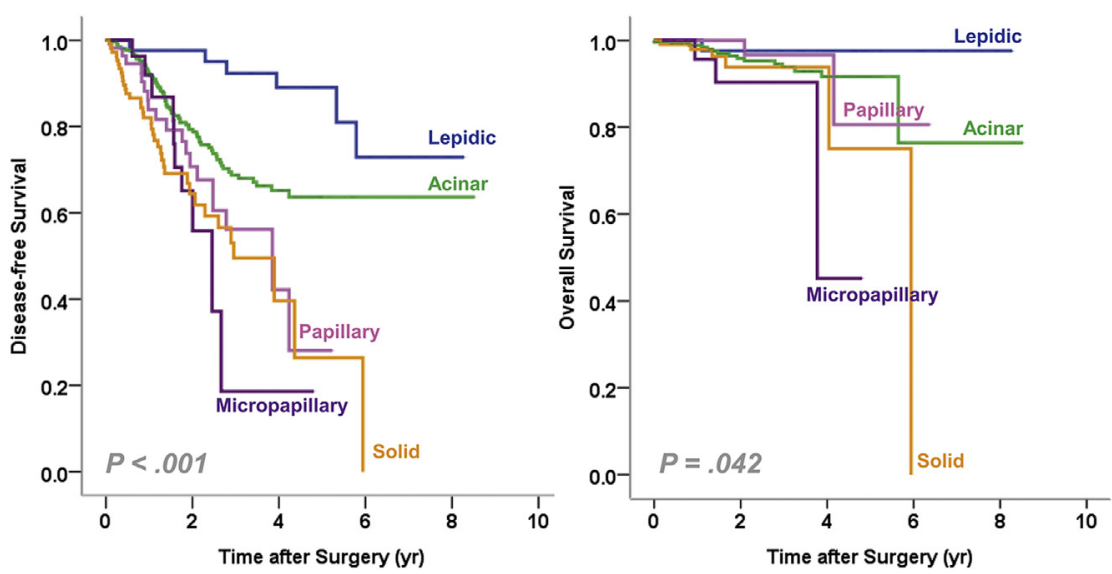

FIGURE 2. Kaplan-Meier survival curves for disease-free and overall survival according to the most predominant subtypes.

\section{Radiologic and Clinical Parameters for Prediction of Micropapillary or Solid Subtype}

The univariate analyses of the clinical and imaging parameters were performed for predicting the presence of the micropapillary or solid subtype on histopathologic examination (Table 3). Among the clinical factors, gender and tumor stage were significantly associated $(P=.03$, and $P<.01$, respectively). Of the $\mathrm{CT}$ findings, large $(P<.01)$, dense $(P=.02)$, low standard deviation of $\mathrm{CT}$ attenuation $(P=.01)$, solid $(P<.01)$, and ill-defined $(P=.04)$ tumors were associated with the presence of the micropapillary or solid subtype. In addition, the SUVmax on PET scans was significantly greater in the tumors with the micropapillary or solid subtype than in those without the subtypes $(P=.04)$.

Multivariate analyses (Table 4) confirmed that greater than stage I (odds ratio [OR], 0.09; 95\% CI, 0.01-0.66; $P=.02)$, tumor size $\geq 2.5 \mathrm{~cm}(\mathrm{OR}, 1.12 ; 95 \% \mathrm{CI}, 1.01$ $1.24 ; P=.02$ ), solid mass (OR, $19.82 ; 95 \% \mathrm{CI}, 1.97$ $199.46 ; P=.01)$, and SUVmax $\geq 7$ (OR, 1.12; 95\% CI, $1.01-1.24 ; P=.03$ ) were independent and significant predictors for the presence of the micropapillary or solid subtype.

We also performed a multivariate analysis of the predictors for tumors containing the micropapillary and solid subtype, separately (Table E1). Tumor size of $\geq 2.5 \mathrm{~cm}(\mathrm{OR}$, $0.86 ; 95 \% \mathrm{CI}, 0.74-0.99 ; P=.04)$ and ill-defined tumor margins (OR, $0.46 ; 95 \%$ CI, $0.25-0.85 ; P=.01$ ) were significant predictors for the presence of the micropapillary subtype. In terms of the solid subtype, male gender (OR, 1.76; 95\% CI, 1.14-2.73; $P=.01$ ), SUVmax $\geq 7$ (OR, 0.93; 95\% CI, 0.88-0.97; $P<.01$ ), and solid mass (OR, 1.73; $95 \%$ CI, $1.07-2.82 ; P=.03$ ) were statistically significant as predictive parameters.

\section{Predictive Probability of Clinical Imaging Parameters for Micropapillary or Solid Subtype}

From our findings, we investigated whether we could accurately identify patients with either the micropapillary or solid subtype by reversely combining 4 clinical imaging predictive factors (Table E2). The 4-factor combination (model 1) of stage 2 or greater, tumor size of $\geq 2.5 \mathrm{~cm}$, SUVmax of $\geq 7$, and the absence of the GGO component was accurate in predicting the presence of the micropapillary or solid subtype, with 34 of $34(100 \%)$ correctly predicting the presence of the micropapillary or solid subtype (OR, 9.73; 95\% CI, 2.35-40.39; $P<.01$ ). Model 2, which included 3 imaging parameters (tumor size, solidity, SUVmax), was also correct in predicting the subtype in 49 of 50 (98\%; OR, 6.04; 95\% CI, 2.57-14.18; $P<.01)$. The area under the ROC curve for model 2 was 0.84 , reflecting good predictability ( $95 \%$ CI, $0.79-0.89 ; P<.01$; Figure E3).

\section{DISCUSSION}

The survival analyses in the present study have demonstrated that the presence of the micropapillary subtype is a statistically significant risk factor for poor OS and DFS. However, the solid subtype showed limited influence on the survival outcomes. The solid subtype played a role as a predictor for poor OS only when coexisting with the micropapillary subtype (Figure E2). This result is analogous to that from the study by Warth and colleagues, ${ }^{5}$ in which the prognosis of solid-predominant ADCs was improved considerably after radiotherapy but the prognosis of micropapillary-predominant ADCs remained poor. In contrast, Solis and colleagues ${ }^{10}$ demonstrated that those with tumors with any solid subtype had lower OS rates than those with tumors without a solid subtype. In our study, the solid and nonsolid groups included $4.3 \%$ (4 of 92) and $9.4 \%$ (11 of 148$)$ with the micropapillary pattern, and this could have been a compounding factor. Therefore, regarding the role of the solid subtype as a predictor of poor survival, additional validation studies focusing on the micropapillary subtype are necessary.

The novel classification of lung ADC has suggested that the pathologic evaluation can be quantified by a $5 \%$ 

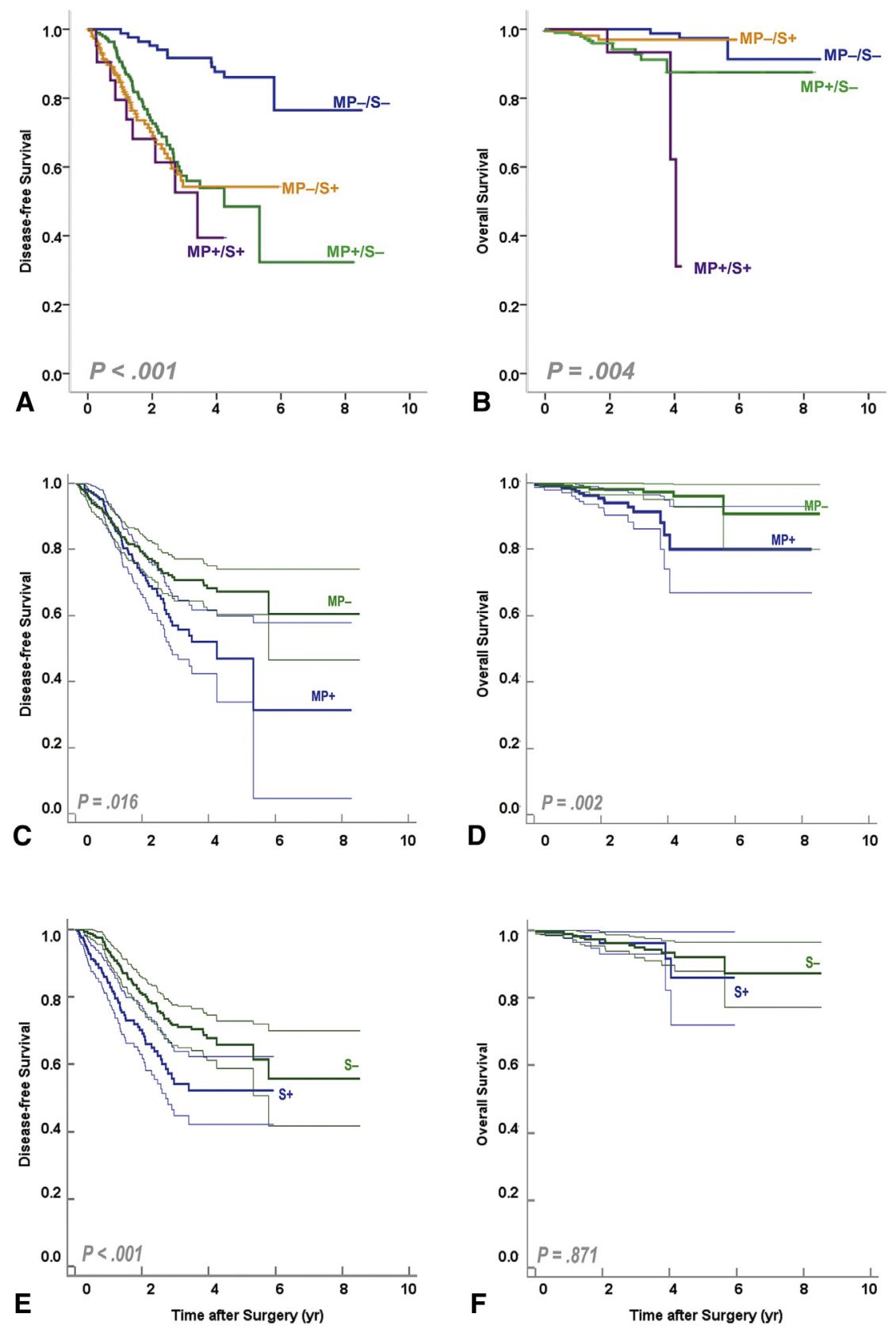

FIGURE 3. Kaplan-Meier survival curves for (A, C, and E) disease-free and (B, D, and F) overall survival according to 4 subgroups of micropapillary ( $M P$ ) and solid $(S)$ subtypes (A and B), presence of micropapillary subtype (C and D), and presence of solid subtype (E and F). C to F, Thin lines indicate $95 \%$ confidence intervals of the survival curves.

increment level for each subtype to sum to $100 \%$ of the whole tumor. ${ }^{1}$ However, we defined the micropapillary or solid subtype as positive when it occupied $\geq 1 \%$ of the entire tumor. This was to determine whether the micropapillary or solid pattern can influence patient survival, if merely present. According to several previous studies, the presence of the micropapillary pattern itself is a poor prognostic factor. ${ }^{9,11}$ The present study has also shown that the presence of $\geq 1 \%$ of the micropapillary subtype is an independent significant factor for a poor prognosis. Considering the clinical significance of the presence of the micropapillary pattern itself, our attempt in the present study to predict the solid or micropapillary subtype using only clinical and imaging factors would have greater clinical implications.

The micropapillary subtype is well-known by its unique growth pattern, not only in the lungs, but also in other organs, such as the breast, urinary bladder, ovary, and major salivary glands. ${ }^{11}$ These tumors with the micropapillary 
TABLE 2. Multivariate analyses for overall survival

\begin{tabular}{lccc}
\hline \multicolumn{1}{c}{ Characteristic } & HR & $\mathbf{9 5} \% \mathbf{C I}$ & $\boldsymbol{P}$ value \\
\hline Gender (male vs female) & 1.42 & $0.58-3.45$ & .44 \\
Age & 1.05 & $0.99-1.09$ & .06 \\
Adjuvant chemotherapy & 1.21 & $0.24-6.18$ & .82 \\
Tumor size & 1.29 & $0.97-1.70$ & .08 \\
Stage (I vs other) & 0.15 & $0.03-0.84$ & $.03^{*}$ \\
Micropapillary pattern & 4.17 & $1.28-13.63$ & $.02 *$ \\
Solid pattern & 1.79 & $0.55-5.81$ & .33 \\
\hline
\end{tabular}

$H R$, Hazard ratio; $C I$, confidence interval. *Statistically significant at $P<.05$.

subtype appear to be invariably associated with a high degree of aggressiveness, manifested by a high stage at presentation, and show massive lymph node and lymphovascular invasion. ${ }^{12}$ Micropapillary ADC consists of small papillary clusters of glandular cells without a fibrovascular core, floating in the airspaces or mucinous pool. ${ }^{1}$ According to Miyoshi and colleagues, ${ }^{13}$ the micropapillary pattern demonstrates disruption of the cell-to-cell adhesion complex, which can act as a basis for its invasive spread.

TABLE 3. Univariate analyses of clinical and imaging parameters for predicting presence of micropapillary or solid subtype

\begin{tabular}{|c|c|c|c|}
\hline Characteristic & $\begin{array}{c}\mathrm{MP}+\text { or } \mathrm{S}+ \\
(\mathrm{n}=\mathbf{4 2 4})\end{array}$ & $\begin{array}{c}M P-\text { and } S_{-} \\
\quad(n=87)\end{array}$ & $\begin{array}{c}P \\
\text { value }\end{array}$ \\
\hline \multicolumn{4}{|l|}{ Clinical factor } \\
\hline Gender & & & $.03 *$ \\
\hline Male & $241(57)$ & $38(44)$ & \\
\hline Female & $183(43)$ & $49(56)$ & \\
\hline Mean age (y) & 60.96 & 59.92 & .42 \\
\hline Stage & & & $<.01 *$ \\
\hline I & $241(57)$ & $80(92)$ & \\
\hline II & $98(23)$ & $6(7)$ & \\
\hline III & $85(20)$ & $1(1)$ & \\
\hline \multicolumn{4}{|l|}{ CT findings } \\
\hline Mean tumor size (mm) & 27.7 & 21.3 & $<.01^{*}$ \\
\hline Mean CT density (HU) & -140.241 & -226.563 & $.02 *$ \\
\hline Mean SD of CT density (HU) & 121.186 & 139.353 & $.01 *$ \\
\hline Tumor solidity & & & $<.01 *$ \\
\hline Solid & $118(28)$ & $8(9)$ & \\
\hline Partly solid & $305(72)$ & $68(78)$ & \\
\hline Not solid (GGO) & $1(0)$ & $11(13)$ & \\
\hline Margin & & & $.04 *$ \\
\hline Well-defined & $367(87)$ & $81(93)$ & \\
\hline Ill-defined & $57(13)$ & $6(7)$ & \\
\hline Spiculation & & & .46 \\
\hline Yes & $133(31)$ & $31(36)$ & \\
\hline No & $291(69)$ & $56(64)$ & \\
\hline Lobulation & & & $.09 *$ \\
\hline Yes & $273(64)$ & $48(55)$ & \\
\hline No & $151(36)$ & $39(45)$ & \\
\hline \multicolumn{4}{|l|}{ PET findings } \\
\hline Mean SUVmax & 8.42 & 3.93 & $<.01 *$ \\
\hline
\end{tabular}

TABLE 4. Multivariate analyses of clinical and imaging parameters for predicting presence of micropapillary or solid subtype

\begin{tabular}{lrlc}
\hline \multicolumn{1}{c}{ Characteristic } & OR & \multicolumn{1}{c}{$\mathbf{9 5} \%$ CI } & $\boldsymbol{P}$ value \\
\hline Gender (male vs female) & 1.49 & $0.83-2.66$ & .18 \\
Stage (I vs other) & 0.09 & $0.01-0.66$ & $.02^{*}$ \\
Tumor size $(\geq 2.5$ vs $<2.5 \mathrm{~cm})$ & 1.12 & $1.01-1.24$ & $.02^{*}$ \\
Tumor solidity (solid vs other) & 19.82 & $1.97-199.46$ & $.01^{*}$ \\
CT density (HU) & 1.00 & $0.99-1.00$ & .60 \\
SD of CT density & 1.00 & $0.99-1.00$ & .35 \\
Margin (well- vs ill-defined) & 0.40 & $0.15-1.08$ & .07 \\
SUVmax ( $\geq 7$ vs $<7$ ) & 1.12 & $1.01-1.24$ & $.03^{*}$ \\
\hline
\end{tabular}

$O R$, Odds ratio; $C I$, confidence interval; $C T$, computed tomography; $H U$, Hounsfield unit; $S D$, standard deviation; SUVmax, maximal standardized uptake value. *Statistically significant at $P<.05$.

Various studies over the decades have been published on lung ADCs with the micropapillary subtype before and after the IASLC/ATS/ERS proposal, and these studies have also established the micropapillary pattern as a distinct poor prognostic marker. ${ }^{11,12,14-16}$ The multivariate analysis of the present study has corroborated those results, proving the micropapillary subtype is a predictor of poor survival, independent of gender, age, adjuvant chemotherapy, and tumor size and stage. Thus, the recommendation of the micropapillary pattern as a new subtype of lung ADC in the novel classification is of great relevance in terms of patient prognosis. In addition, the micropapillary subtype was observed in $44.6 \%$ of resected lung ADC specimens in our institution. This subtype has been encountered quite frequently in lung ADCs. Similar results were reported previously by other investigators. Roh and colleagues ${ }^{17}$ reported that the micropapillary subtype and nodal micrometastasis were found in $16(45.7 \%)$ and 14 (40\%) of 35 lung ADC specimens, respectively.

The identification of poor prognostic histologic subtypes using only clinical and imaging factors is of great importance for predicting patient prognosis and determining the treatment plan for inoperable and operable patients. ${ }^{18}$ The novel classification might influence the selection of patients in need of adjuvant therapy; therefore, imaging biomarkers would be of great help in determining the preoperative therapeutic plan. The prediction of a poor prognostic histologic subtype is also important for patients with unresectable lung cancer, because their pathologic information is solely dependent on small pieces of specimen from biopsies and not from a resected specimen. Moreover, approximately $80 \%$ of patients with lung cancer will have unresectable tumor at the initial presentation. The results of the multivariate analysis of the present study suggested that stage greater than I, tumor size of $\geq 2.5 \mathrm{~cm}$ in diameter, solid mass without a GGO component, and SUVmax of $\geq 7$ were independent and significant predictors for the presence of the micropapillary or solid subtype.

Our study had several limitations. First, our study was a retrospective study performed at a single institution. Our 
results might have been influenced by the patient population. However, we tried to include a large cohort with a logical selection process to minimize the selection bias. Second, the range of follow-up time after surgery was relatively wide. This might have influenced patient prognosis. Selecting a proper follow-up period is important. Third, the tumor size was limited to $3 \mathrm{~cm}$ in diameter. This was because pathologic examination of the whole mass will be limited if the tumor is $>3 \mathrm{~cm}$. However, the tumor size restriction can also influence patient prognosis and radiologic parameters. Fourth, we used the same data set to test the models in the supplementary analysis, and the results might have been overoptimistic. However, in the absence of a data set for true external validation, it was our best option to present the probability of the predictors. Finally, by subgrouping the patients into 4 groups according to the minimal (1\%) presence of each ADC subtype, the meaning of each subtype proportion within the tumor might have been mitigated. However, again, the patient prognosis does not depend solely on the histopathologic examination findings. It should be determined by integrating the histopathologic findings, tumor stage, tumor size, attenuation value, and imaging biomarker study (eg, PET) results.

In conclusion, the present study has demonstrated that both micropapillary and solid patterns, defined in the novel IASLC/ATS/ERS classification, are risk factors for predicting poor DFS and that the micropapillary pattern is a single powerful prognostic factor for OS. In addition, tumor stage greater than I, lesion size of $\geq 2.5 \mathrm{~cm}$, pure solid nature without GGO, and SUVmax of $\geq 7$ were significant parameters for predicting the presence of a micropapillary or solid subtype. Thus, the evaluation of these factors can allow clinicians to have more accurate indicators of prognosis preoperatively and, therefore, might facilitate selecting patients in need of more aggressive therapy, although they have low-stage disease.

\section{References}

1. Travis WD, Brambilla E, Noguchi M, Nicholson AG, Geisinger KR, Yatabe Y, et al. International Association for the Study of Lung Cancer/American Thoracic Society/European Respiratory Society international multidisciplinary classification of lung adenocarcinoma. J Thorac Oncol. 2011;6:244-85.

2. Lee HY, Jeong JY, Lee KS, Kim HJ, Han J, Kim BT, et al. Solitary pulmonary nodular lung adenocarcinoma: correlation of histopathologic scoring and patient survival with imaging biomarkers. Radiology. 2012;264:884-93.
3. Yoshizawa A, Motoi N, Riely GJ, Sima CS, Gerald WL, Kris MG, et al. Impact of proposed IASLC/ATS/ERS classification of lung adenocarcinoma: prognostic subgroups and implications for further revision of staging based on analysis of 514 stage I cases. Mod Pathol. 2011;24:653-64.

4. Russell PA, Wainer Z, Wright GM, Daniels M, Conron M, Williams RA. Does lung adenocarcinoma subtype predict patient survival? A clinicopathologic study based on the new International Association for the Study of Lung Cancer/American Thoracic Society/European Respiratory Society international multidisciplinary lung adenocarcinoma classification. J Thorac Oncol. 2011; 6:1496-504.

5. Warth A, Muley T, Meister M, Stenzinger A, Thomas M, Schirmacher P, et al. The novel histologic International Association for the Study of Lung Cancer/ American Thoracic Society/European Respiratory Society classification system of lung adenocarcinoma is a stage-independent predictor of survival. J Clin Oncol. 2012;30:1438-46.

6. Okada M, Tauchi S, Iwanaga K, Mimura T, Kitamura Y, Watanabe H, et al. Associations among bronchioloalveolar carcinoma components, positron emission tomographic and computed tomographic findings, and malignant behavior in small lung adenocarcinomas. J Thorac Cardiovasc Surg. 2007;133:1448-54.

7. Travis WD, Brambilla E, Muller-Hermelink HK, Haris CC. World Health Organization classification of tumors: pathology and genetics. In: Tumours of the Lung, Pleura, Thymus and Heart. Lyon, France: IARC Press; 2004.

8. Lee HY, Han J, Lee KS, Koo JH, Jeong SY, Kim BT, et al. Lung adenocarcinoma as a solitary pulmonary nodule: prognostic determinants of CT, PET, and histopathologic findings. Lung Cancer. 2009;66:379-85.

9. Ohe M, Yokose T, Sakuma Y, Miyagi Y, Okamoto N, Osanai S, et al. Stromal micropapillary component as a novel unfavorable prognostic factor of lung adenocarcinoma. Diagn Pathol. 2012;7:3.

10. Solis LM, Behrens C, Raso MG, Lin HY, Kadara H, Yuan P, et al. Histologic patterns and molecular characteristics of lung adenocarcinoma associated with clinical outcome. Cancer. 2012;118:2889-99.

11. Maeda R, Isowa N, Onuma H, Miura H, Harada T, Touge H, et al. Lung adenocarcinomas with micropapillary components. Gen Thorac Cardiovasc Surg. 2009;57:534-9.

12. Nassar H. Carcinomas with micropapillary morphology: clinical significance and current concepts. Adv Anat Pathol. 2004;11:297-303.

13. Miyoshi T, Shirakusa T, Ishikawa Y, Iwasaki A, Shiraishi T, Makimoto Y, et al. Possible mechanism of metastasis in lung adenocarcinomas with a micropapillary pattern. Pathol Int. 2005;55:419-24.

14. Amin MB, Tamboli P, Merchant SH, Ordonez NG, Ro J, Ayala AG, et al. Micropapillary component in lung adenocarcinoma: a distinctive histologic feature with possible prognostic significance. Am J Surg Pathol. 2002;26:358-64.

15. Makimoto Y, Nabeshima K, Iwasaki H, Miyoshi T, Enatsu S, Shiraishi T, et al. Micropapillary pattern: a distinct pathological marker to subclassify tumours with a significantly poor prognosis within small peripheral lung adenocarcinoma $(</=20 \mathrm{~mm})$ with mixed bronchioloalveolar and invasive subtypes (Noguchi's type C tumours). Histopathology. 2005;46:677-84.

16. Kadota K, Colovos C, Suzuki K, Rizk NP, Dunphy MP, Zabor EC, et al. FDGPET SUVmax combined with IASLC/ATS/ERS histologic classification improves the prognostic stratification of patients with stage I lung adenocarcinoma. Ann Surg Oncol. 2012;19:3598-605.

17. Roh MS, Lee JI, Choi PJ, Hong YS. Relationship between micropapillary component and micrometastasis in the regional lymph nodes of patients with stage I lung adenocarcinoma. Histopathology. 2004;45:580-6.

18. Tsutani Y, Miyata Y, Mimae T, Kushitani K, Takeshima Y, Yoshimura M, et al. The prognostic role of pathologic invasive component size, excluding lepidic growth, in stage I lung adenocarcinoma. J Thorac Cardiovasc Surg. 2013;146: $580-5$. 


\section{APPENDIX E1. CT AND PET/CT ACQUISITION}

Helical CT images were obtained with an 8-detector row (LightSpeed Ultra, GE Healthcare) or 16-detector row (LightSpeed16, GE Healthcare) CT scanner. Contrast-enhanced CT scanning was obtained 90 seconds after the administration of contrast material $(100 \mathrm{~mL}$ of iopamidol, Iomeron 300, Bracco, Milan, Italy) at a rate of $1.5 \mathrm{~mL} / \mathrm{s}$ using a power injector. This was followed by $20-\mathrm{mL}$ saline flushing at a rate of $1.5 \mathrm{~mL} / \mathrm{s}$. Contrast-enhanced CT images were obtained with the following parameters: detector collimation, $0.625 \mathrm{~mm}$; field of view, $34.5 \mathrm{~cm}$; beam pitch, 1.35 or 1.375 ; gantry speed, 0.6 second per rotation; $120 \mathrm{kVp} ; 150$ to $200 \mathrm{~mA}$; and section thickness, $1.25 \mathrm{~mm}$ for transverse images. All imaging data were reconstructed using soft tissue algorithms.

Fluorodeoxyglucose PET-CT images were acquired using a PET-CT device (Discovery LS; GE Healthcare, Milwaukee, Wis), which consisted of a PET scanner (Advance NXi, GE Healthcare) and an 8-section CT scanner (Light-Speed Plus, GE Healthcare).

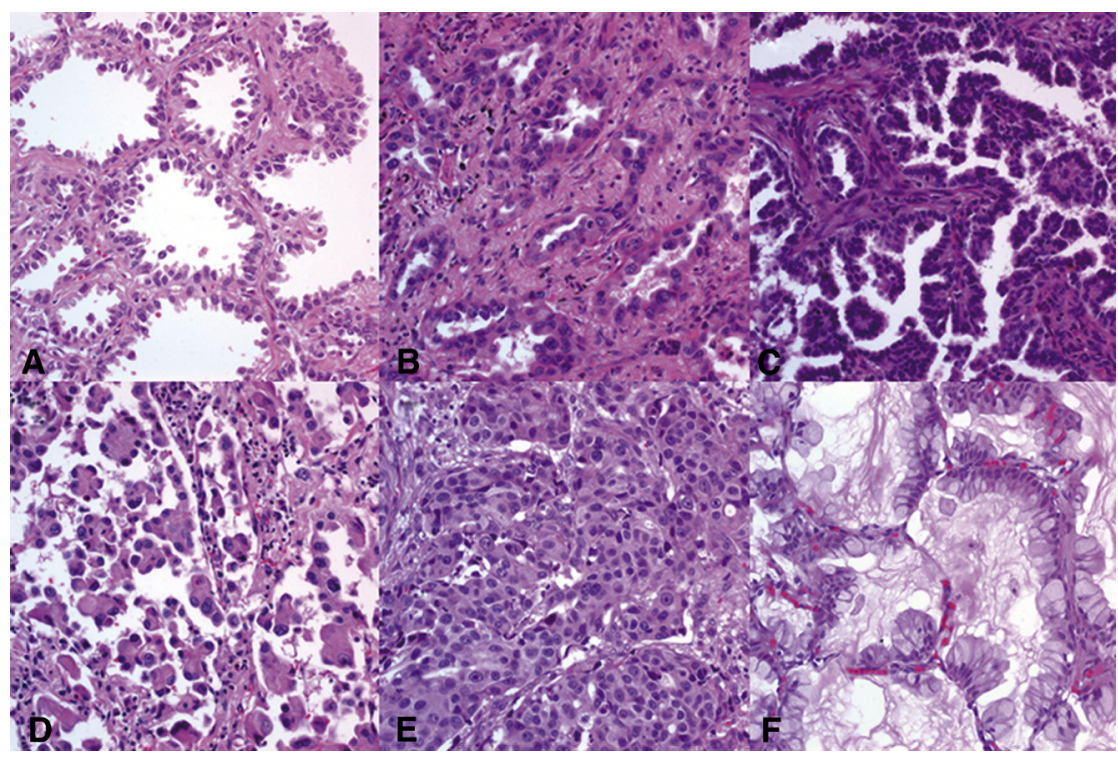

FIGURE E1. Representative photomicrographs of the 6 histologic patterns: A, lepidic; B, acinar; C, papillary; D, micropapillary; E, solid; and F, mucinous (hematoxylin-eosin stain, original magnification $\times 400$ ).
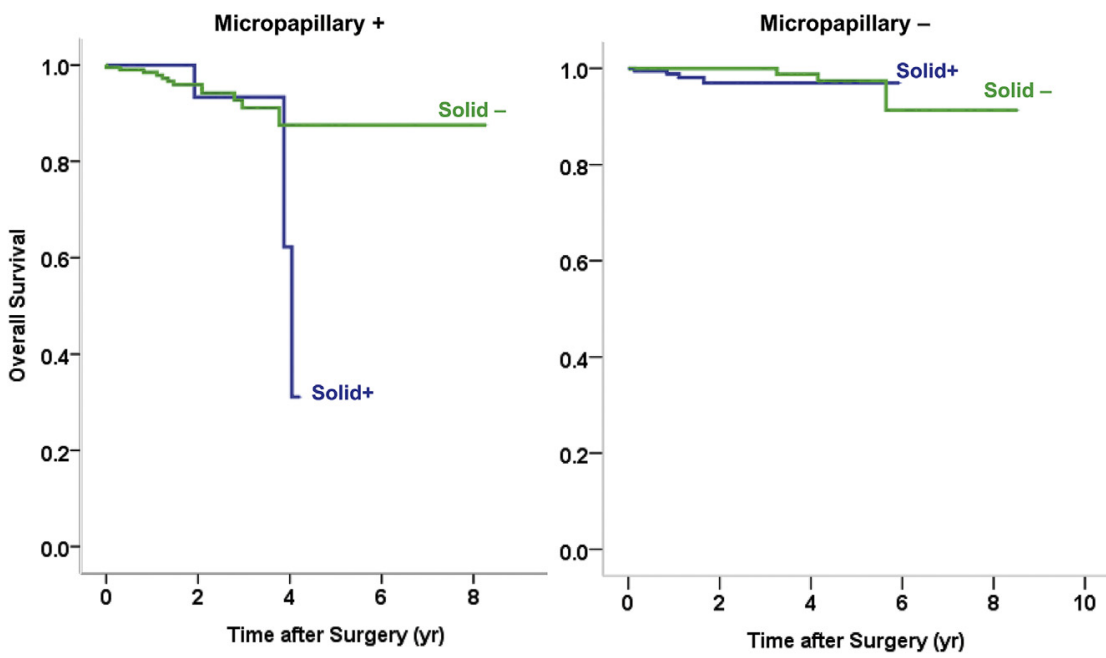

FIGURE E2. Subgroup analysis stratified by micropapillary subtype on overall survival. 


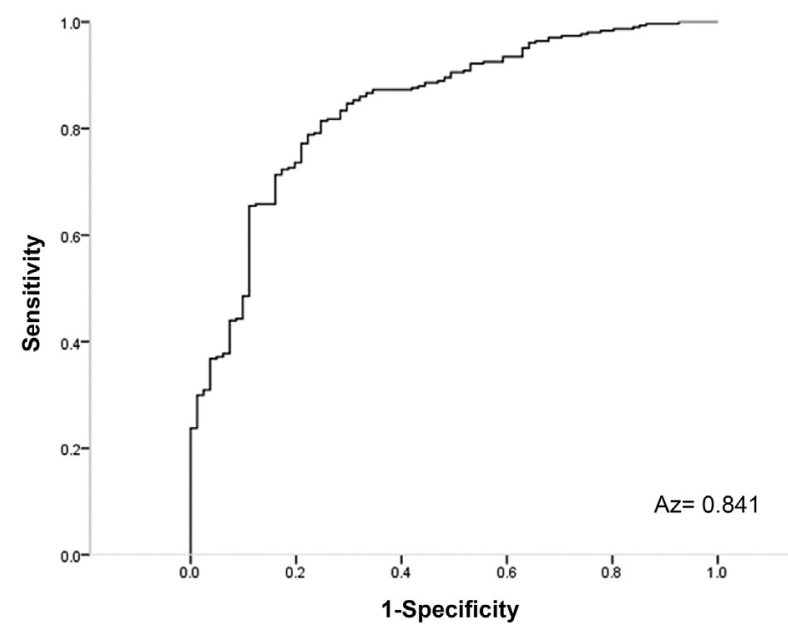

FIGURE E3. Receiver operating characteristic curve of model 2 of tumor size of $\geq 2.5 \mathrm{~cm}$, maximal standard uptake value of $\geq 7$, and the absence of ground glass opacity component, plotting sensitivity versus 1 - specificity, illustrating an area under the curve of 0.84 . AZ, Area under the receiver operating characteristic curve.
TABLE E1. Multivariate analyses of respective clinical and imaging parameters for predicting presence of each micropapillary and solid subtype

\begin{tabular}{lccr}
\hline \multicolumn{1}{c}{ Characteristics } & OR & $\mathbf{9 5} \%$ CI & $\boldsymbol{P}$ value* \\
\hline Presence of micropapillary component & & & \\
$\quad$ Tumor size $(\geq 2.5$ vs $<2.5 \mathrm{~cm})$ & 0.86 & $0.74-0.99$ & .04 \\
$\quad$ Margin (well- vs ill-defined) & 0.46 & $0.25-0.85$ & .01 \\
Presence of solid component & & & \\
Gender (male vs female) & 1.76 & $1.14-2.73$ & .01 \\
SUVmax ( $\geq 7$ vs $<7$ ) & 0.93 & $0.88-0.97$ & $<.01$ \\
Tumor solidity (solid vs other) & 1.73 & $1.07-2.82$ & .03 \\
\hline
\end{tabular}

$O R$, Odds ratio; $C I$, confidence interval; SUVmax, maximal standardized uptake value. *All statistically significant at $P<.05$.

TABLE E2. Predictivity of tumors containing micropapillary or solid subtype

\begin{tabular}{|c|c|c|c|c|c|c|}
\hline Model & Factors & All patients* (n) & Patients with MP or $\mathbf{S}$ subtype $\dagger$ (n) & OR $(95 \%$ CI $)$ & $P$ value & $\operatorname{AUC}(95 \% \mathbf{C I})$ \\
\hline 1 & $\begin{array}{l}\text { Stage } \geq 2 \\
\text { SUVmax } \geq 7 \\
\text { Size } \geq 2.5 \mathrm{~cm} \\
\text { Solid without GGO component }\end{array}$ & 34 & 34 & $9.73(2.35-40.39)$ & .002 & $0.945(0.923-0.968)$ \\
\hline 2 & $\begin{array}{l}\text { SUVmax } \geq 7 \\
\text { Size } \geq 2.5 \mathrm{~cm} \\
\text { Solid without GGO component }\end{array}$ & 50 & 49 & $6.04(2.57-14.18)$ & $<.001$ & $0.841(0.794-0.889)$ \\
\hline 3 & $\begin{array}{l}\text { SUVmax } \geq 7 \\
\text { Size } \geq 2.5 \mathrm{~cm}\end{array}$ & 131 & 130 & $1.79(1.02-3.04)$ & .037 & $0.880(0.848-0.912)$ \\
\hline
\end{tabular}

$M P$, Micropapillary; $S$, solid; $O R$, odds ratio; $C I$, confidence interval; $A U C$, area under the receiver operating characteristic curve; $S U V m a x$, maximal standardized uptake value; $G G O$, ground glass opacity. *Patients who fit into each model. †Patients with micropapillary or solid pattern of total given in "All patients." 